\title{
Assessment of the Radiological Changes Involving Articular Surfaces of Temporomandibular Joints in Patients with Osteoarthritis and Rheumatoid Arthritis using Computed Tomography
}

\section{Edara Naga Priyanka' ${ }^{1}$, Nalli Prasanth Kumar ${ }^{1}$, Sankar Narayana Sarma G ${ }^{2}$, Kusuma Kumari Donepudi ${ }^{3}$, Srinivas Prasad Vijayan ${ }^{4}$, Kolappan $\mathbf{R}^{5}$, Anitha Krishnan Pandarathodiyil ${ }^{6 *}$}

${ }^{1}$ Department of Oral Medicine and Radiology, Lenora Institute of Dental Sciences, Rajahmundry, Andhra Pradesh, India

${ }^{2}$ Department of Periodontics, GSL Dental College Rajahmundry, Andhra Pradesh, India

${ }^{3}$ Private Dental Practitioner, Welfare Dental Clinic, Ravulapalem, Andhra Pradesh, India

${ }^{4}$ Department of Prosthodontics and Restorative Dentistry, School of Dentistry, College of Medicine and Health Sciences, University of Rwanda, Remera Campus, Kigali, Rwanda

${ }^{5}$ Department of Oral Surgery, Kusum Devi Sunderlal Dugar Jain Dental College and Hospital, Kolkata, West Bengal, India

${ }^{6}$ Faculty of Dentistry, SEGi University, Jalan Teknologi, Kota Damansara, Petaling Jaya, Selangor, Malaysia

*Corresponding Author: Anitha Krishnan Pandarathodiyil, Faculty of Dentistry, SEGi University, Jalan Teknologi, Kota Damansara, Petaling Jaya, Selangor, Malaysia.
Received: August 02, 2021

Published: August 19, 2021

(C) All rights are reserved by Edara Naga

Priyanka., et al.

\section{Abstract}

Background: Temporomandibular joint (TMJ) disorders refer to a complex set of conditions; manifested by pain in the area of the jaws and associated muscles and limitations in their ability to make normal movements like swallowing, mastication, and speech. Arthritis also affects the TMJ.

Aim: The purpose of the present study was to assess the radiological changes involving the TMJ of patients with osteoarthritis (OA) and rheumatoid arthritis (RA) using computed tomography (CT) scans for early detection and disease management.

Materials and Methods: The study group comprised of 30 patients between 18 and 70 years of age, who were randomly selected from patients visiting the orthopaedics and rheumatology department of a government institution. The patients were categorized into two groups based on the type of arthritis. All subjects underwent radiographic investigations and CT scans. Additionally, biochemical blood test for the presence of RA factor, and digital radiographs of the long bones were done for all patients.

Results: The female to male ratio for RA was 6.5:1 and for OA 3.2:1. In RA, the pathological findings were present in all age groups, and irregular joint space was the most predominant finding on both sides. In OA, the involvement was seen in the elderly age group, with a predominant finding of irregular joint space and flattening of articular surface on the right side and erosion of condyle and articular eminence space on the left side. Statistically significant values $[\mathrm{P}=0.002]$ for flattening of articular surface on left side were obtained.

Conclusion: CT study showed positive evidence of TMJ involvement in both types of arthritis. Thus, we conclude that CT scan would serve as a valuable tool for detecting TMJ disorders early and securing better treatment outcomes.

Keywords: Temporomandibular Disorders; Rheumatoid Arthritis; Osteoarthritis; Computed Tomography; Articular Surface 
Assessment of the Radiological Changes Involving Articular Surfaces of Temporomandibular Joints in Patients with Osteoarthritis and

\section{Introduction}

Bone is a highly specialized tissue, characterized by rigidity, hardness and the power of regeneration and repair [1]. Similar to all other connective tissues, it contains ground substance, fibres and cells. Bone is classified as a specialized connective tissue because of presence of minerals (calcium salts) in its intracellular matrix [2]. Bone constantly undergoes remodelling and reshaping during life to help it adapt to changing biomechanical forces, as well as to remove old, micro damaged bone and replace it with new, mechanically stronger bone to help preserve strength [3]. A joint or articulation (or articular surface) is the connection between bones in the body which link the skeletal system as a functional whole. They allow for different degrees and types of movements. Formation of the temporomandibular joints (TMJ) occurs at around 12 weeks of gestational period, when the joint spaces and the articular disc develop. At approximately 10 weeks of intrauterine life, the component of the future joint becomes evident in the mesenchyme between condylar cartilage of the mandible and the developing temporal bone [4]. The TMJs are the two joints connecting the jawbone to the skull. It is a bilateral synovial articulation between the temporal bone of the skull above and the mandible below. It is from these bones the name has been derived. TMJ is categorized as a "ginglymoarthrodial" joint since it is both a ginglymus (hinging joint) and an arthrodial (sliding) joint. The condyle of the mandible articulates with the temporal bone in the mandibular fossa. The mandibular fossa is a concave depression in the squamous portion of the temporal bone [5]. This joint allows the protrusion, elevation, retraction, and lateral sliding movements of the mandible, which are mainly produced by masticatory muscles. There are diseases that compromise TMJ and adjacent structures, constituting temporomandibular disorders (TMD). They are characterized by pain, joint sounds, chewing and speech impairments, irregular jaw function and even changes in global posture [6]. Although not life-threatening, they can be detrimental to the quality of life in individuals [7].

Rheumatoid arthritis (RA) is a chronic, systemic, autoimmune inflammatory disorder characterized by joint inflammation, erosive properties and symmetric multiple joint involvement [8]. TMJ problems are present in about more than $50 \%$ of patients of RA. TMJ is usually the last joint to be involved and is associated with many clinical signs and symptoms, like pain, inflammation, limited movements, swelling (joint stiffness), and muscle spasms [9]. TMJ is very rarely affected in the early phase of the disease, thus posing diagnostic challenges for the dentist [8]. Osteoarthritis (OA) is a chronic, progressive, and debilitating disease, defined by the gradual deterioration (degeneration) of the cartilage in a joint. TMJ osteoarthritis (TMJ-OA) is more frequent in females and its prevalence increases in relation to age. Many factors such as overloading, bruxism, unilateral chewing, genetic factors and internal derangement are held responsible for the development of TMJ-OA [10]. OA is an age-related disease, and the WHO estimates that globally $25 \%$ of adults aged over 65 years suffer from pain and disability associated with this disease. The percentage of TMJ-OA in age group 9 - 90 years range from $28 \%$ to $38 \%$ and incidence increases with advancing age [11]. The cardinal features of TMJ-OA are both clinical and radiographic. The clinical features are tenderness in the joint region, pain on movement of the joint during mouth opening and lateral excursion, and hard grating or crepitus. Radiographic signs of the disease are cortical bone erosion, flattening of joint compartments with productive bone changes such as sclerosis and osteophyte formation. These signs of TMJ-OA represent different stages of the disease process. Erosive lesions and joint space narrowing indicate acute or early change, whereas sclerosis, flattening, subchondral cyst, and osteophyte formation may indicate late changes in TMDs [11].

Radiographic examination of the TMJ is indicated when there are clinical signs of pathological conditions, mainly bony changes that may influence the diagnosis and treatment planning. Conventional radiographs fail to show the early lesions due to their obvious limitations. Hence, CT has been largely used for the assessment of TMJ anatomy. This provides better visualization of the joint's bony structures, allowing detection of small changes, which are undetectable through conventional radiographs due to the anatomy of the region, superimpositions, and presence of overlapped structures. Multi-slice CT allows obtaining thinner slices and high image quality in less acquisition time. In multi-slice CT, multiple overlapping slices can be reconstructed from a single examination permitting higher quality reconstructed images without additional patient irradiation [12]. It has minimal superimposition of structures and allows better visualization. CT proved to be extremely useful for evaluating joints such as the hip and shoulder. These joints are often difficult to examine with conventional radiographic methods because of their complex anatomic structures. The same is true of the TMJ [13]. Hence, the present study was conducted to evaluate the incidence of osseous changes in the TMJ of patients afflicted with RA and OA using CT to detect the disorders in their early phase.

\section{Aims of the Study}

1. To evaluate the osseous changes of TMJ in RA and OA patients using CT scans.

2. To detect the presence of narrowing of the joint space, irregular joint space, flattening of articular surfaces, osteophyte 
Assessment of the Radiological Changes Involving Articular Surfaces of Temporomandibular Joints in Patients with Osteoarthritis and Rheumatoid Arthritis using Computed Tomography

formation, anterior lipping of the condyle, subchondral cyst, erosions of condyle and articular eminence in RA and OA patients using CT.

\section{Methodology and Data Collection}

Ethical approval and informed consent were obtained from the Institutional Ethical Committee (IEC) serial No: CEC/12/2015-16 of St. Joseph Dental College and Hospital, Eluru, India. After explaining the study procedure to the subjects, written, and signed informed consents were taken from the participants before the commencement of the study. Patients above 18 years of age (both males and females) who were diagnosed with RA or OA and willing to participate were included in the study. Pregnant and lactating women, patients needing ambulatory assistance, patients with systemic diseases other than OA and RA, diabetes, and hypertension, were excluded from the study. Thirty patients of both genders from 18 to 70 years of age participated in the study. Fifteen patients were afflicted with OA [Group-A] while the other fifteen had RA [Group-B]. After biochemical investigation for RA factor for RA patients and a plain radiograph of the long bones for OA patients, CT imaging of the TMJs were taken. CT scan was taken for the direct axial view and coronal views, and reconstructed to sagittal planes with contiguous slice thickness of $2 \mathrm{~mm}$.

For CT exposure, the scans were carried out on TOSHIBA Asteion single slice VP. Scans were carried out with $120 \mathrm{Kvp}$ and $180 \mathrm{~mA}$ with acquisition time of 1.5 seconds/slice. For radiographic interpretation, the following devices were used: i. Radiant Dicom viewer 4.0.3.16415 [64-bit], 6/11/2.17 software and ii. Lenovo G50 personal computer (Intel inside core i3).

The selected subjects were instructed to remove any removable dental appliances and metal objects that would likely obstruct the radiation passage through the head and neck region. Then they were made to lie down on the CT couch in supine position, and head was supported on a head rest with a FOV (Field of View) of $250 \mathrm{~mm}$ using slice thickness of $2 \mathrm{~mm}$. The helical images were reformatted and images were then used for CT measurements of osseous changes in TMJ. All the images were evaluated to detect the presence of narrowing and irregular joint space, flattening of articular surfaces, osteophyte formation, anterior lipping of the condyle, subchondral cyst, erosions of condyle and articular eminence and sclerosis (Figure 1-7).

\section{Data analysis}

The values obtained were recorded and entered in the proforma sheet specially designed for the study. Data collected was subjected to statistical analysis using statistical package for social sciences (SPSS) version 21, SPSS.inc, Chicago 1989 - 2007. Statisti-

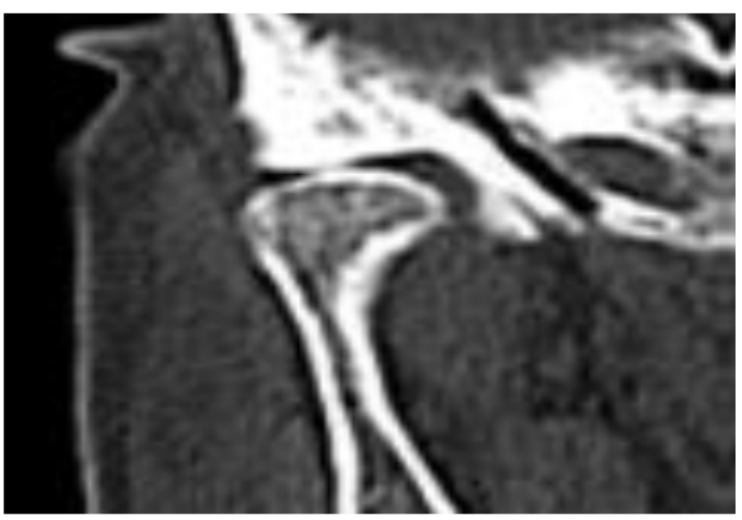

Figure 1: Narrowing of joint space.

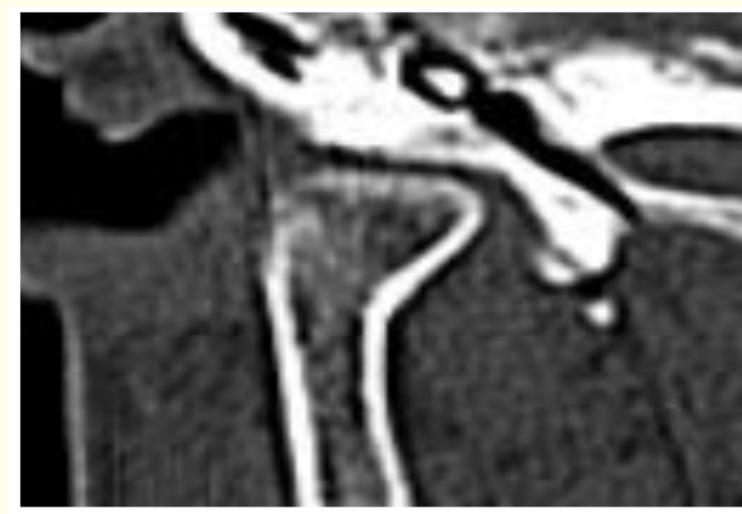

Figure 2: Flattening of joint space.

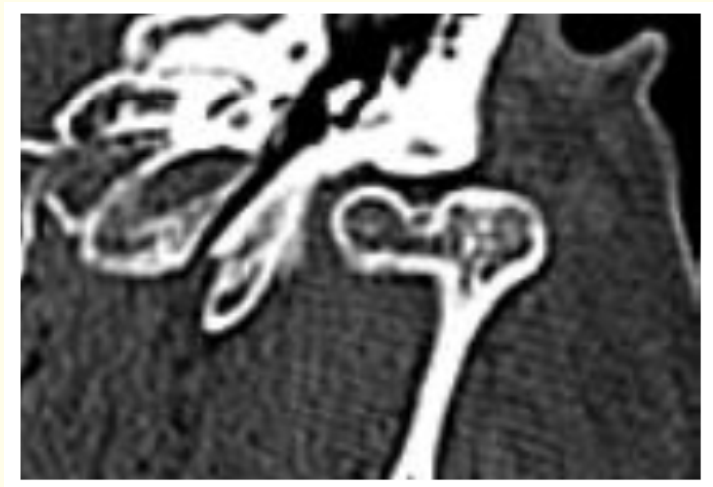

Figure 3: Erosion of condylar and articular eminence. 


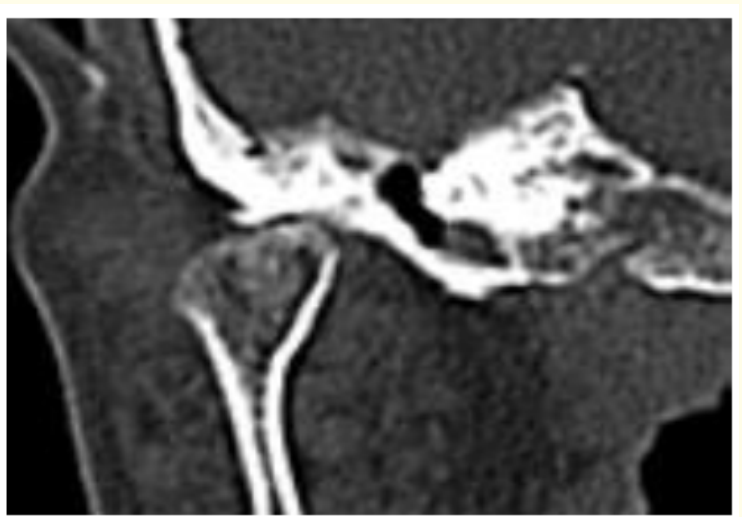

Figure 4: Sclerosis.

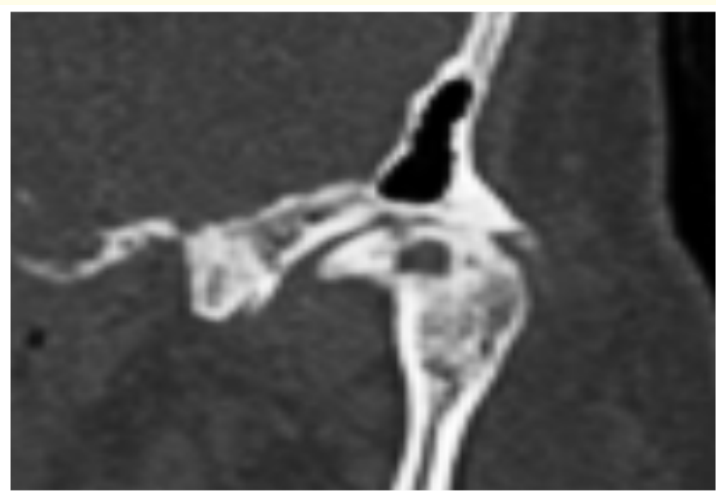

Figure 5: Subchondral cyst.

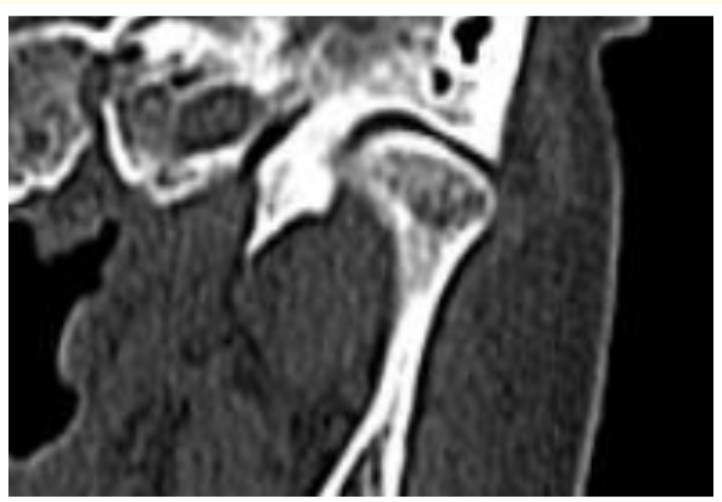

Figure 6: Osteophyte formation.

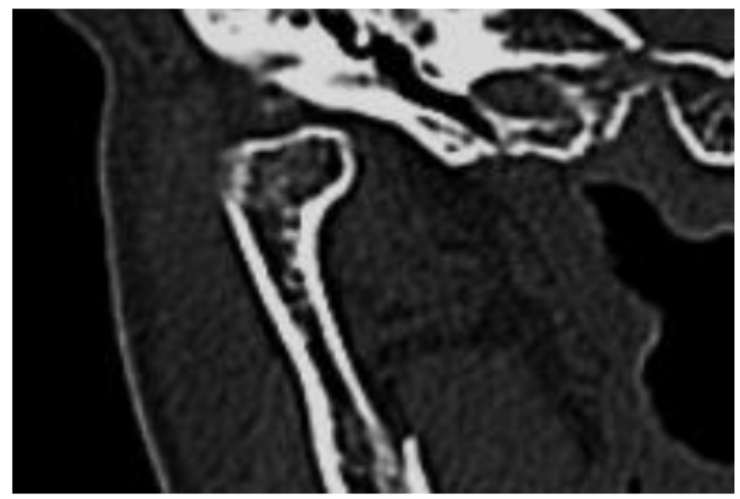

Figure 7: Irregular joint space.

cal significance was set at 0.05 , which indicates that any $\mathrm{p}$ value less than 0.05 will be considered significant. Chi square tests were done to evaluate the osseous changes of TMJ in RA and OA patients using CT. Sixteen radiographs were randomly selected. These radiographs were evaluated for intra observer agreement on two occasions with a one-month interval between evaluations. Kappa values were calculated to know the intra observer variability.

\section{Results}

The comparative evaluation of CT scan changes in the TMJs of OA and RA patients was carried out. Out of fifteen RA patients, two were males and thirteen were females and the fifteen $\mathrm{OA}$ patients comprised of five males and ten females.

Out of fifteen patients in the RA group, the right side was involved in four of those patients, with two patients exhibiting left side involvement. Bilateral involvement was noted in nine patients. Among the fifteen patients in the OA group, the right side was involved in five of those patients with no left side involvement. For eight patients, bilateral involvement was observed with two patients exhibiting normal joint function. The presence of osseous changes in RA and OA on right and left sides of TMJ were evaluated. These changes are shown in table 1 . Similarly, the osseous findings on the right \& left sides of the TMJ in both the genders in RA and OA were studied and are shown in table 2 and 3 respectively.

The prevalence of RA and OA among different age groups has also been evaluated. In the RA group, 5 subjects were between the age group of 41-50 years, 3 subjects were seen in each of the 3 age groups [ 31 - 40 years, 41 - 50 years and above 60 years] and 1 subject below 30 years, was observed. In the OA group, 7 subjects were seen between the age group of 51 to 60 years, 5 subjects 
Assessment of the Radiological Changes Involving Articular Surfaces of Temporomandibular Joints in Patients with Osteoarthritis and Rheumatoid Arthritis using Computed Tomography

\begin{tabular}{|l|c|c|c|c|c|c|c|c|}
\hline \multirow{2}{*}{ Findings } & \multicolumn{3}{|c}{ Rheumatoid Arthritis } & \multicolumn{4}{c|}{ Osteoarthritis } \\
\cline { 2 - 9 } & Right & Left & Total & p-valve & Right & Left & Total & p-value \\
\hline Narrowing of joint space & $4(23.5 \%)$ & $2(13.3 \%)$ & 6 & 0.361 & $2(10 \%)$ & $3(17.6 \%)$ & 5 & 0.624 \\
\hline Irregular joint space & $6(35.5 \%)$ & $4(26.6 \%)$ & 10 & 0.439 & $5(25 \%)$ & $3(17.6 \%)$ & 8 & 0.409 \\
\hline Flattening of articular surfaces & $3(17.6 \%)$ & $3(20.3 \%)$ & 6 & 1.0 & $5(25 \%)$ & $3(17.6 \%)$ & 8 & 0.409 \\
\hline Osteophyte formation & $0(0.0 \%)$ & $0(0.0 \%)$ & 0 & - & $2(10 \%)$ & $2(11.7 \%)$ & 4 & 1.00 \\
\hline Anterior lipping of condyle & $0(0.0 \%)$ & $0(0.0 \%)$ & 0 & - & $0(0.0 \%)$ & $0(0.0 \%)$ & 0 & - \\
\hline Sub chondral cyst & $1(5.8 \%)$ & $1(6.6 \%)$ & 2 & 1.0 & $0(0.0 \%)$ & $0(0.0 \%)$ & 0 & - \\
\hline $\begin{array}{l}\text { Erosion of condylar and articular } \\
\text { eminence }\end{array}$ & $3(17.6 \%)$ & $4(26.6 \%)$ & 7 & 0.666 & $4(20 \%)$ & $4(23.8 \%)$ & 8 & 1.00 \\
\hline Sclerosis & $0(0.0 \%)$ & $1(6.6 \%)$ & 1 & 0309 & $2(10 \%)$ & $2(11.7 \%)$ & 4 & 1.00 \\
\hline Total & $17(100 \%)$ & $15(100 \%)$ & 32 & & $20(100 \%)$ & $17(100 \%)$ & 37 & \\
\hline
\end{tabular}

Table 1: Showing the presence of osseous changes in Rheumatoid arthritis and Osteoarthritis on right and left sides of the TMJ.

\begin{tabular}{|c|c|c|c|c|c|c|c|c|}
\hline \multirow{3}{*}{ Findings } & \multicolumn{8}{|c|}{ Rheumatoid arthritis } \\
\hline & \multicolumn{4}{|c|}{ Right } & \multicolumn{4}{|c|}{ Left } \\
\hline & Male & Female & Total & p-valve & Male & Female & Total & p-value \\
\hline Narrowing of joint space & $0(0.0 \%)$ & $4(25 \%)$ & 4 & 0.360 & $0(0.0 \%)$ & $2(15.8 \%)$ & 2 & 0.551 \\
\hline Irregular joint space & $0(0.0 \%)$ & $6(37.5 \%)$ & 6 & 0.215 & $0(0.0 \%)$ & $4(30.7 \%)$ & 4 & 0.360 \\
\hline Flattening of articular surfaces & $1(100 \%)$ & $2(12.5 \%)$ & 3 & 0.255 & $2(100 \%)$ & $1(7.6 \%)$ & 3 & 0.002 \\
\hline Osteophyte formation & $0(0.0 \%)$ & $0(0.0 \%)$ & 0 & - & $0(0.0 \%)$ & $0(0.0 \%)$ & 0 & - \\
\hline Anterior lipping of condyle & $0(0.0 \%)$ & $0(0.0 \%)$ & 0 & - & $0(0.0 \%)$ & $0(0.0 \%)$ & 0 & - \\
\hline Sub chondral cyst & $0(0.0 \%)$ & $1(6.25 \%)$ & 1 & 0.685 & $0(0.0 \%)$ & $1(7.6 \%)$ & 1 & 0.685 \\
\hline $\begin{array}{l}\text { Erosion of condylar and articular } \\
\text { eminence }\end{array}$ & $0(0.0 \%)$ & $3(18.75 \%)$ & 3 & 1.00 & $0(0.0 \%)$ & $4(30.7 \%)$ & 4 & 0.360 \\
\hline Sclerosis & $0(0.0 \%)$ & $0(0.0 \%)$ & 0 & - & $0(0.0 \%)$ & $1(7.6 \%)$ & 1 & 0.685 \\
\hline Total & $1(100 \%)$ & $16(100 \%)$ & 17 & & $2(100 \%)$ & $13(100 \%)$ & 15 & \\
\hline
\end{tabular}

Table 2: Showing osseous findings on right and left sides in both the genders in rheumatoid arthritis.

\begin{tabular}{|c|c|c|c|c|c|c|c|c|}
\hline \multirow[t]{3}{*}{ Findings } & \multicolumn{8}{|c|}{ Osteoarthritis } \\
\hline & \multicolumn{4}{|c|}{ Right } & \multicolumn{4}{|c|}{ Left } \\
\hline & Male & Female & Total & p-value & Male & Female & Total & p-valve \\
\hline Narrowing of joint space & $0(0.0 \%)$ & $2(16.6 \%)$ & 2 & 0.283 & $1(14.2 \%)$ & $2(20 \%)$ & 3 & 1.00 \\
\hline Irregular joint space & $2(25 \%)$ & $3(25.1 \%)$ & 5 & 0.699 & $2(28.7 \%)$ & $1(10 \%)$ & 3 & 0.171 \\
\hline Flattening of articular surfaces & $3(37.5 \%)$ & $2(16.6 \%)$ & 5 & 0.121 & $2(28.7 \%)$ & $1(10 \%)$ & 3 & 0.171 \\
\hline Osteophyte formation & $0(0.0 \%)$ & $2(25.1 \%)$ & 2 & 0.283 & $0(0.0 \%)$ & $2(20 \%)$ & 2 & 0.283 \\
\hline Anterior lipping of condyle & $0(0.0 \%)$ & $0(0.0 \%)$ & 0 & - & $0(0.0 \%)$ & $0(0.0 \%)$ & 0 & - \\
\hline Sub chondral cyst & $0(0.0 \%)$ & $0(0.0 \%)$ & 0 & - & $0(0.0 \%)$ & $0(0.0 \%)$ & 0 & - \\
\hline $\begin{array}{l}\text { Erosion of condylar and articular } \\
\text { eminence }\end{array}$ & $1(12.5 \%)$ & $3(25.1 \%)$ & 4 & 0.680 & $1(14.2 \%)$ & $3(30 \%)$ & 4 & 0.680 \\
\hline Sclerosis & $2(25 \%)$ & $0(0.0 \%)$ & 2 & 0.032 & $1(14.2 \%)$ & $1(10 \%)$ & 2 & 0.591 \\
\hline Total & $8(100 \%)$ & $12(100 \%)$ & 20 & & $7(100 \%)$ & $10(100 \%)$ & 17 & \\
\hline
\end{tabular}

Table 3: Showing distribution of osseous findings on right and left sides of the TMJ in both the genders in osteoarthritis. 
Assessment of the Radiological Changes Involving Articular Surfaces of Temporomandibular Joints in Patients with Osteoarthritis and

were seen above 61 years and 3 were seen in the 31 - 40 years age group. When evaluated for presence of certain finding in different age groups of patients afflicted with RA on right and left sides, a total of 32 findings were observed. 17 were observed on the right side and 15 on the left. More number of pathological findings [10] were noted in the age group between 51 - 60 years, followed by the age group of 60 years where involvement was observed in 8 joints. 7 joints showed pathological findings in the age group of $31-40$ years and 6 joints in the age group of 41 - 50 years. The least [ 1 joint] pathological findings were seen in the below 30 age group. Predominant findings like irregular joint space were observed in 10 joints, distributed in all age groups on the right side. On the left side, erosion of condylar and articular eminence was detected on 7 joints between the age groups of 31 to 60 years, followed by narrowing of joint space and flattening of articular surfaces in 6 joints. The highest prevalence of irregular joint space $[3$ joints $=2$ right joints +1 left joint] was observed in the age group that was above 60 years. Flattening of articular surfaces was completely absent in age groups below 40 years. Osteophyte formation and anterior lipping of the condyle were not uniformly evident on all RA patients. Subchondral cyst was only evident in the 51-60 age group [1 right joint +1 left joint].

When evaluated for presence of certain finding in different age groups of patients afflicted with OA on right and left sides, a total of 35 findings were observed, out of which 18 were detected on the right side and 17 on the left side. More number of findings were observed in the age group above 60 years [ 14 findings] followed by 13 findings in the 51 - 60 age group. Eight findings were detected in the 31 - 40 years age group, and no findings were detected in the following age groups: below 30 years and 41 - 50 years. The most common findings like irregular joint space, flattening of articular surfaces and erosion of condylar and articular eminence were observed in 8 joints on both the right and left sides, followed by narrowing of joint space in 5 joints, sclerosis in 4 joints and osteophyte formation in 2 joints. The highest prevalence of narrowing of joint space was observed in $51-60$ years age group with a predominant left side presentation [ 1 right +2 left joints]. Irregular joint space was more prevalent in the $31-40$ [2 right joints +1 left joint] age group and above 60 years age group [ 2 right joints +1 left joint]. Flattening of condyle was a common presentation in patients above 60 years of age [ 2 right joints +2 left joint]. Intriguingly, osteophyte formation was observed in 2 left joints on patients above 60 years of age. Anterior lipping of the condyle and subchondral cyst were completely absent in all age groups. Erosion of condyle and articular eminence were highly prevalent in the 51 - 60 years age group [2 right joints +2 left joint] and Sclerosis was evenly distributed [1 right joint +1 left joint] in the $31-40$ years age group.
All the radiographs were evaluated by one assessor on two occasions with a one-month interval between the two evaluations. The evaluations were checked for intra observer agreement, and the Kappa values were calculated to assess the intra observer variability as shown in table 4 .

\begin{tabular}{|l|c|c|c|}
\hline $\begin{array}{l}\text { S. } \\
\text { no }\end{array}$ & Findings & $\begin{array}{c}\text { Kappa } \\
\text { value }\end{array}$ & \\
\hline 1 & Narrowing of joint space & 0.846 & $\begin{array}{c}\text { Almost perfect } \\
\text { agreement }\end{array}$ \\
\hline 2 & Irregular joint space & 0.818 & $\begin{array}{c}\text { Almost perfect } \\
\text { agreement }\end{array}$ \\
\hline 3 & $\begin{array}{c}\text { Flattening of articular } \\
\text { surfaces }\end{array}$ & 0.673 & Substantial \\
\hline 4 & $\begin{array}{c}\text { Erosion of condylar and } \\
\text { articular eminence }\end{array}$ & 1 & $\begin{array}{c}\text { Almost perfect } \\
\text { agreement }\end{array}$ \\
\hline 5 & Sclerosis & 0.765 & Substantial \\
\hline
\end{tabular}

Table 4: Table showing kappa values for intra observer variability.

\section{Discussion}

RA is the most common inflammatory disease of the joints. It is an immune-mediated systemic disease of the young and middle-aged adults characterized by proliferative and destructive changes in the synovial membrane and the articular and the peri-articular structures. Eventually, joints are destroyed, fibrosed, or ankylosed. It causes widespread vasculitis of the small arterioles. It can affect any joint in the body. However, it involves the peripheral joints more often and rarely affects the larger joints [14]. According to Lee DY, Kim YJ, Song YH, Lee NH., et al. [15], the criteria for determination of the type of condylar bony changes are narrowing of joint space, osteophyte formation, flattening of condylar head, subchondral cyst, erosions of condyle and articular eminence and sclerosis.

OA has been considered as an age-related degenerative change of the articular cartilage and the subchondral bone in synovial joints including TMJ, leading to pain and disability [16]. De Leeuw., et al. [17], stated that of the disorders afflicting the TMJ, OA, and internal derangement are frequently observed. It is stated that progressive nature of internal derangement in several consecutive stages will eventually lead to radiographically visible degenerative changes, which may be extensive. Controversy exists that OA can also lead to disc displacement. The present study was carried out to find the various bony changes in the TMJs of elderly patients afflicted with $\mathrm{OA}$ and $\mathrm{RA}$, so that initiating treatment during early stages of the ailment may prevent disease progression. OA and RA both have different etiopathogeneses but affect the TM Joints simi- 
Assessment of the Radiological Changes Involving Articular Surfaces of Temporomandibular Joints in Patients with Osteoarthritis and

larly from a clinically and radiological perspective. CT is one of the preferred imaging techniques used for the detection of alteration in hard tissues such as osseous changes in the condyles and temporal components as well as the other articulating and non-articulating surfaces in patients with TMJ diseases.

In the present study, the most commonly affected age group in RA is 41 - 50 years, which was almost similar to the observations made by Buduru., et al. [14] who found the highest prevalence in age group of $50 \pm 11$ years. It is also close to the findings of the study conducted by Ardic., et al. [18] [mean age to be 46 years] and Oynther and Tronje., et al. [19] [44 years]. The pathological findings were observed in all the RA patients [100\%]. Similar results were observed in the study by Buduru., et al. [14] where they found $90 \%$ of cases with pathological findings. In the present study, the most predominant finding on the right side was irregular joint space $(40 \%)$, followed by narrowing of joint space (23.5\%), flattening of articular surfaces (17.6\%) and erosion of condylar and articular eminence (17.6\%). This is somewhat similar to study conducted by Buduru., et al. [14] where, narrowing of joint space was a predominant finding followed by flattening of articular surface and osteophyte formation. Interestingly, our study did not observe any osteophyte formation in RA patients, which was in contradictory to Celiker, et al. [20] who found osteophyte formation in 70\%, reduced joint space in $70 \%$ and erosions in $60 \%$ of cases. In our study, the prevalence of sub chondral cyst was least in RA patients [6.6\%], which was in accordance with studies done by Tamer Gheita., et al. [21], Deoghare A., et al [22]. Voog U., et al. [23] observed erosions in $50 \%$ of cases. But interestingly, only $23.1 \%$ of cases were observed erosions in the present study.

In the RA group, the most predominant finding seen on the left side was irregular joint space (26.7\%), and erosion of condylar and articular eminence $(26.7 \%)$ followed by flattening of articular surfaces $(20 \%)$, narrowing of joint space (13.3\%), and sub chondral cyst and sclerosis (6.6\%). This is similar to study conducted by Tamer Gheita., et al. [21] and Deoghare A., et al [22].

In $\mathrm{OA}$, the most commonly affected age group is 51 - 60 years, a finding which was similar to the observations made by Buduru., et al. [14] [49 \pm 15 years]. Voog., et al. [23] and Toller [24] found the mean age of occurrence of $\mathrm{OA}$ in their studies at 42 years and 40 years respectively, which was on the lower side. FM Mani., et al. [11] observed a generalized presentation of OA in the age group of 50 - 80 years with mean age of 63.06 years which is on the higher side. These findings are in accordance with the American College of Rheumatology criteria for the diagnosis of OA where the age range is described to be above 50 years. In OA group, the most predomi- nant findings seen on the right side of the joint was irregular joint space (25\%) and flattening of articular surfaces (25\%) followed by erosion of condylar and articular eminence (20\%), narrowing of joint space $(10 \%)$, sclerosis $(10 \%)$ and osteophyte formation $(10 \%)$. These findings are similar to the studies by Deoghare A., et al. [22] in their study where out of 15 OA cases, they found flattening in $43.33 \%$, osteophyte formation in $26.6 \%$. In their study on OA patients, Buduru., et al. [14] found narrowing of joint space in $75 \%$ of cases, osteophyte formation in $55 \%$ of patients and flattening of articular surface in $30 \%$ of cases. Osteophyte formation was found to be higher. Oynther and Tronje., et al. [19] in their study on OA, found flattening of condylar surface in $40 \%$ of the cases, sclerosis in 35\% of cases and osteophyte formation in 55\% of them. There are other studies which showed contradictory results (FM Mani., et al. [11] where out of 10 females and 5 males of OA cases; they found erosion in $56.6 \%$ and joint space narrowing in $40 \%$ of cases. Toller [24] in his study found erosion in $93.3 \%$ and osteophyte formation in $21 \%$ of the cases. In the OA group, the most predominant finding on the left side was erosion of condylar and articular eminence (26.7\%), narrowing of joint space (17.6\%), irregular joint space $(17.6 \%)$, followed by sclerosis and osteophyte formation (11.7\%). And there are studies which showed erosion on lower side like studies done by Oynther and Tronje., et al. [19] and Deoghare., et al [22].

While comparing the findings of RA and OA, OA involves a few joints whereas RA involves multiple joints with the TMJ being one of the joints to be involved frequently. In general, OA involves joints unilaterally while RA usually involves joints bilaterally. In contrast, the present study showed $53.3 \%$ of TMJ's involved bilaterally in OA, a finding which is close to study conducted by Buduru., et al [14].

In the present study, TMJs of $60 \%$ patients in RA group were involved bilaterally. This finding is similar to the findings conducted by Buduru., et al. [14], Goupille., et al. [25] and Holmlund., et al [26]. OA and RA have always been affecting females more commonly than males which was confirmed in our study again where female to male ratio obtained in RA group was 6.5:1 and in OA group it was 2:1, a total of 4.25:1 respectively. Various studies done by Wiberg., et al. [27], Ardic., et al. [18] and Oynther., et al. [19] have proved that females might be predisposed to dysfunctional remodeling of the TMJ's. This female preponderance for dysfunctional remodeling of the TMJs suggests a potential role of sex hormones (i.e. estrogen, prolactin) as modulators of this response. This is the first study in which irregular joint space has been detected as an osseous change in RA and OA patients, and interestingly this pathological finding was found to be highly prevalent in both RA and 
Assessment of the Radiological Changes Involving Articular Surfaces of Temporomandibular Joints in Patients with Osteoarthritis and

OA groups. Anterior lipping of the condyle is also a new parameter which has been included in this study. But there were no cases reported with these findings in the samples taken.

\section{Summary and Conclusion}

Detection of early changes in the TMJs of patients afflicted with RA and OA patients has assumed considerable significance and garnered sufficient scientific attention to prevent impairment of the functional capacity of the entire masticatory system. This usually remains undetected until the changes become symptomatic and hinder the normal life style of the patients. There are various clinical and radiological methods to detect these osseous changes in the TMJ. One of the most widely acknowledged and effective methods in this regard is the use of CT scans. CT scans have proven to be the most important and widely used diagnostic tool in detecting the various osseous changes of the TMJ. It gives a detailed view in all sections to assess disease activity, which helps the clinician to plan treatment strategies and monitor therapeutic responses. The present study was conducted in RA and OA patients to detect the early osseous changes for initiation of treatment protocols and to avoid further joint discomfort. In the present study, a total of 30 patients [60 joints], of which 15 patients with RA and 15 patients with OA were taken. We found a higher prevalence of osseous changes of patients suffering from RA ranging from 18 to 70 years. Our study also supported a higher prevalence of osseous changes in $\mathrm{OA}$ in the elderly age group. Therefore, a thorough assessment of the TMJs is recommended for all patients above the age of 40 , who are afflicted with generalized joint pain and in cases where osseous involvement of the TMJs is suspected. Therefore, we opine and conclude that CT scans give us valuable information in detecting osseous changes of the TMJ at the early stages of disease progression which helps the clinician to formulate effective treatment strategies so as to prevent further damage to the joints.

\section{Conflict of Interest}

The authors declare no conflict of interest.

\section{Bibliography}

1. Kini U and Nandeesh B N. "Physiology of bone formation, remodeling and metabolism".

2. Pal GP. Textbook of histology". $3^{\text {rd }}$ edition (2012).

3. Whiting William Charles and Rugg Dynatomy, Stuart Dynamic Human Anatomy 10 (2006): 40.

4. Salentijn L. "Biology of Mineralized Tissues Prenatal Skull Development". Columbia University College of Dental Medicine post-graduate dental lecture series (2007).
5. Alomar X., et al. "Anatomy of the temporomandibular joint". Seminars in Ultrasound, CT, and MR 28.3 (2007): 170-183.

6. Chaves PJ., et al. "Incidence of postural changes and temporomandibular disorders in students". Acta Ortopedica Brasileira 25.4 (2017).

7. Shi Z., et al. "Hyaluronate for temporomandibular joint disorders". The Cochrane Database of Systematic Reviews (2003): 1.

8. Sodhi A., et al. "Rheumatoid arthritis affecting temporomandibular joint". Contemporary Clinical Dentistry 6.1 (2015): 124-127.

9. Ruparelia PB., et al. "Bilateral TMJ involvement in rheumatoid arthritis". Case Reports in Dentistry (2014): 262430.

10. Kilic SC and Kilic N. "Temporomandibular joint osteoarthritis: cone beam computed tomography findings, clinical features, and correlations". International Journal of Oral and Maxillofacial Surgery 44.10 (2015): 1268-1274.

11. Mani FM and Sivasubramanian SS. "A study of temporomandibular joint osteoarthritis using computed tomographic imaging". Biomedical Journal 39 (2016): 201-206.

12. Cara AC. "Validity of single- and multislice CT for assessment of mandibular condyle lesions". Dent Maxillofacial Radiology 36 (2007): 24-27.

13. Clyde A., et al. "Computed Tomography of the Temporomandibular Joint Meniscus". Journal of Oral and Maxillofacial Surgery 41 (1983): 512-517.

14. Buduru K., et al. "Assessment of radiological changes involving the articular surface of the temporomandibular joint in patients with osteoarthritis and rheumatoid arthritis using computed tomography scan: A prospective clinico-radiological study". Archives of Medical Science 5 (2017): 24-29.

15. Lee DY., et al. "Comparison of bony changes between panoramic radiograph and cone beam computed tomographic images in patients with temporomandibular joint disorders". Korean Journal of Orthodontics 40.6 (2010): 364-372.

16. Yamada K., et al. "Observation of three cases of temporomandibular joint osteoarthritis and mandibular morphology during adolescence using helical CT". Journal of Oral Rehabilitation 31 (2004): 298e305.

17. De Leeuw R., et al. "Radiographic signs of temporomandibular joint osteoarthrosis and internal derangement 30 years after

Citation: Edara Naga Priyanka, Nalli Prasanth Kumar, Sankar Narayana Sarma G, Kusuma Kumari Donepudi, Srinivas Prasad Vijayan, Kolappan R, Anitha Krishnan Pandarathodiyil. "Assessment of the Radiological Changes Involving Articular Surfaces of Temporomandibular Joints in Patients with Osteoarthritis and Rheumatoid Arthritis using Computed Tomography". Acta Scientific Dental Sciences 5.9 (2021): $95-103$. 
nonsurgical treatment". Oral Surgery, Oral Medicine, Oral Pathology, Oral Radiology, and Endodontology 79 (1995): 382e92.

18. Ardic F., et al. "The comprehensive evaluation of temporomandibular disorders seen in rheumatoid arthritis". Australian Dental Journal 51.1 (2006): 23-28.

19. Oynther GW., et al. "Radiographic changes in the temporomandibular joint in patients with generalized osteoarthritis and rheumatoid arthritis". Oral Surgery, Oral Medicine, Oral Pathology, Oral Radiology, and Endodontology 81 (1996): 613-618.

20. Celiker R., et al. "Temporomandibular Joint Involvement in Rheumatoid Arthriis". Scandinavian Journal of Rheumatology 24 (1995): 22-25.

21. Gheita T., et al. "A Using Clinical and Multislice Computer Tomographic Features to Assess Temporomandibular Joint Osseous Involvement in Rheumatoid Arthritis: A Preliminary Study". Turkish Journal of Rheumatology's 27.1 (2012): 47-55.

22. Deoghare A and Shirish S Degwekar. "Clinical and CT scan Evaluation of Temporomandibular Joints with Osteoarthritis and Rheumatoid Arthritis". Journal of Indian Academy of Oral Medicine and Radiology 22.4 (2010): S1-5.

23. Voog U., et al. "Inflammatory mediators and radiographic changes in temporomandibular joints of patients with rheumatoid arthritis". Acta Odontologica Scandinavica 61 (2003): 57-64.

24. Toller PA. "Osteoarthrosis of the mandibular condyle". The British Dental Journal 134 (1973): 223-231.

25. Goupille P., et al. "Direct coronal computed tomography of the temporomandibular joint in patients with rheumatoid arthritis". The British Journal of Radiology 65 (1992): 955-960.

26. Holmlund AB., et al. "Rheumatoid arthritis and disk derangement of the temporomandibular joint. A comparative arthroscopic study". Oral Surgery, Oral Medicine, Oral Pathology, Oral Radiology, and Endodontology 73 (1992): 273-277.

27. Wiberg B and Wänman A. "Signs of osteoarthrosis of the temporomandibular joints in young patients: A clinical and radiographic study". Oral Surgery, Oral Medicine, Oral Pathology, Oral Radiology, and Endodontology 86 (1998): 158-164.

Volume 5 Issue 9 September 2021

(C) All rights are reserved by Edara Naga Priyanka.,

et al. 